\title{
SECOND ORDER SYSTEMS WITH NONLINEAR BOUNDARY CONDITIONS
}

\author{
WALTER G. KELLEY
}

\begin{abstract}
Some existence theorems are established for nonlinear second order systems with nonlinear two point boundary conditions. The system is assumed to satisfy certain differential inequality conditions at the boundary of a region.
\end{abstract}

Let $R^{n}$ be $n$-dimensional Euclidean space with scalar product $x \cdot y$ for $x$ and $y$ in $R^{n}$ and norm $\|x\|=\sqrt{x \cdot x}$. In the following discussion, $f:[a, b] \times R^{n}$ $\times R^{n} \rightarrow R^{n}$, where $[a, b]$ is an interval of real numbers, will be assumed continuous. The system

$$
x^{\prime \prime}=f\left(t, x, x^{\prime}\right),
$$

will be considered together with boundary conditions of the form

$$
\begin{aligned}
& x(a)-A_{1} x^{\prime}(a)=0, \\
& x(b)+A_{2} x^{\prime}(b)=0,
\end{aligned}
$$

or

$$
\begin{aligned}
& B_{1} x(a)-x^{\prime}(a)=0, \\
& B_{2} x(b)+x^{\prime}(b)=0,
\end{aligned}
$$

where $A_{1}, A_{2}, B_{1}, B_{2}$ are continuous functions from $R^{n}$ to $R^{n}$.

Lasota and Yorke [3] have proved existence theorems for (1), (2), (3) in the case where $A_{1}$ and $A_{2}$ are linear and positive definite, using Lyapunov-type inequalities for $f$. We show in this paper that similar results are true under suitable conditions on the boundary functions even if these functions are nonlinear and differential inequality conditions on $f$ are assumed to hold only at the boundary of a region. Nonlinear boundary conditions have been studied in the scalar case by a variety of authors, including Bebernes and Wilhelmsen [1], and Jackson and Klaasen [2].

Suppose $r: R^{n} \rightarrow R$ is of class $C^{2}$ with gradient $u: R^{n} \rightarrow R^{n}$ and Hessian

Received by the editors March 25, 1976.

AMS (MOS) subject classifications (1970). Primary 34B15.

Key words and phrases. Nonlinear system, nonlinear two point boundary value problem.

- American Mathematical Society 1977 
$P: R^{n} \rightarrow \mathfrak{L}\left(R^{n}, R^{n}\right)$. Let the first and second derivatives of $r$ with respect to (1) be denoted by

$$
r^{\prime}=u \cdot x^{\prime}, \quad r_{f}^{\prime \prime}=x^{\prime} P \cdot x^{\prime}+u \cdot f
$$

Auxiliary functions of this type have been used by Mawhin [4] to study (1), (2), (3) in the case $A_{1}=A_{2}=0$.

We say that $f$ satisfies condition $(\mathrm{N})$ with respect to $r$ if there is a positive, nondecreasing, continuous function $\phi$ defined on $(0, \infty)$ such that $s^{2} / \phi(s)$ $\rightarrow \infty$ as $s \rightarrow \infty$ and $\|f(t, x, y)\| \leqslant \phi(\|y\|)$ for $t \in[a, b], r(x) \leqslant 0, y \in R^{n}$. This is a Nagumo condition on $f$ of the type used by Schmitt and Thompson [5].

THEOREM 1. Let $r$ be as above and assume $\{x: r(x)<0\}$ is bounded and contains 0 . Suppose $u(x) \neq 0$ whenever $r(x)=0$ and

$$
\inf \{(u(x) \cdot x) /\|u(x)\|\|x\|: r(x)=0\}=\alpha \geqslant 0 .
$$

Assume $f$ satisfies condition $(\mathrm{N})$ with respect to $r$ and

$$
r_{f}^{\prime \prime} \geqslant 0 \text { when } r=0 \text { and } r^{\prime}=0 .
$$

Suppose $\left\{y: r\left(A_{1} y\right)<0\right\}$ is bounded and contains 0 , and

$$
\begin{gathered}
\quad \inf \left\{\left(y \cdot A_{1} y\right) /\|y\|\left\|A_{1} y\right\|: r\left(A_{1} y\right)=0\right\} \geqslant \sqrt{1-\alpha^{2}}, \\
\text { if } D=\left\{y: r\left(-A_{2} y\right)=0 \text {, or } r\left(A_{1} y\right)=0 \text { and } A_{2} y \neq 0\right\} \neq \varnothing, \\
\text { then } \inf \left\{\left(y \cdot A_{2} y\right) /\|y\|\left\|A_{2} y\right\|: y \in D\right\}>\sqrt{1-\alpha^{2}} .
\end{gathered}
$$

Then there is at least one solution $x$ of (1), (2), (3) with $r(x(t)) \leqslant 0$ for $t \in[a, b]$.

Proof. We assume initially that $f$ satisfies

$$
r_{f}^{\prime \prime}>0 \text { when } r=0 \text { and } r^{\prime}=0
$$

and that initial value problems for (1) have unique solutions.

Define $W=\left\{(x, y) \in R^{n} \times R^{n}: x=A_{1} y\right\}$ and $V=\{(x, y) \in W: r(x)$ $<0\}$. Note that $V \neq \varnothing$ since $\left(A_{1} 0,0\right) \in V$. We now define a function $T: \bar{V} \rightarrow R^{n}$. If $(x, y) \in \bar{V}$, then there is a unique solution $x(t)$ of (1) with $\left(x(a), x^{\prime}(a)\right)=(x, y)$. Since by Lemma 2.1 of [5] the derivative $x^{\prime}(t)$ is bounded as long as $r(x(t)) \leqslant 0$, the extension theorem for ordinary differential equations implies that the trajectory $\left(t, x(t), x^{\prime}(t)\right)$ extends to the boundary of $[a, b] \times\{x: r(x) \leqslant 0\} \times R^{n}$. Let $t_{0}$ be the smallest value of $t$ for which $r(x(t))=0$, or, if there is no such $t$, let $t_{0}=b$. Define $T(x, y)=x\left(t_{0}\right)$ $+A_{2} x^{\prime}\left(t_{0}\right)$.

We claim that $T$ is continuous on $\bar{V}$. Let $(x, y) \in \bar{V}$ and $\left(x_{k}, y_{k}\right) \in \bar{V}$ for $k=1,2, \ldots$, and suppose $\left(x_{k}, y_{k}\right) \rightarrow(x, y)$ as $k \rightarrow \infty$. Let $x$ and $x_{k}$ be the 
solutions of (1) with $\left(x(a), x^{\prime}(a)\right)=(x, y)$ and $\left(x_{k}(a), x_{k}^{\prime}(a)\right)=\left(x_{k}, y_{k}\right)$ for $k=1,2, \ldots$.

Suppose $r(x(t))<0$ for $t \in[a, b]$. By the standard convergence theorem, $r\left(x_{k}(t)\right)<0$ for $k$ sufficiently large and $t \in[a, b]$. Since $\left(x_{k}(b), x_{k}^{\prime}(b)\right)$ $\rightarrow\left(x(b), x^{\prime}(b)\right)$ as $k \rightarrow \infty$, we conclude $T\left(x_{k}, y_{k}\right) \rightarrow T(x, y)$ as $k \rightarrow \infty$ in this case.

Otherwise, there is a smallest $t_{0}$ so that $r\left(x\left(t_{0}\right)\right)=0$. Suppose $a<t_{0}<b$. Let $s(t)=r(x(t))$ for all $t$ in the domain of $x$. Since $s(t)<0$ for $a \leqslant t<t_{0}$ and $s\left(t_{0}\right)=0$, we have $s^{\prime}\left(t_{0}\right) \geqslant 0$. If $s^{\prime}\left(t_{0}\right)>0$, there is a $\delta>0$ so that $s(t)>0$ for $t_{0}<t \leqslant t_{0}+\delta$. If $s^{\prime}\left(t_{0}\right)=0$, then by (7)' $s^{\prime \prime}\left(t_{0}\right)>0$. This is impossible because $s(t)<0$ for $a \leqslant t<t_{0}$.

Fix $\lambda \in(0, \delta)$. Let

$$
\varepsilon=\inf \left\{|s(t)|: t \in\left[0, t_{0}-\lambda\right] \cup\left[t_{0}+\lambda, t_{0}+\delta\right]\right\} .
$$

Choose $k$ sufficiently large so that $\left|r(x(t))-r\left(x_{k}(t)\right)\right|<\varepsilon / 2,\left\|x_{k}(t)-x(t)\right\|$ $<\lambda$ and $\left\|x_{k}^{\prime}(t)-x^{\prime}(t)\right\|<\lambda$ for $t \in\left[0, t_{0}+\delta\right]$. For these values of $k, r\left(x_{k}(t)\right)$ $<-\varepsilon / 2<0$ for $t \in\left[0, t_{0}-\lambda\right]$ and $r\left(x_{k}(t)\right)>\varepsilon / 2>0$ for $t \in\left[t_{0}+\lambda, t_{0}\right.$ $+\delta$ ]. Hence for each such $k$ there is a smallest $t$, say $t_{k}$, with $r\left(x_{k}\left(t_{k}\right)\right)=0$ and $t_{k} \in\left(t_{0}-\lambda, t_{0}+\lambda\right)$. Furthermore, $\left\|x_{k}\left(t_{k}\right)-x\left(t_{0}\right)\right\|$ and $\left\|x_{k}^{\prime}\left(t_{k}\right)-x^{\prime}\left(t_{0}\right)\right\|$ are no larger than the diameter of the set

$$
\left\{(t, x, y): t_{0}-\lambda<t<t_{0}+\lambda,\|x-x(t)\|<\lambda,\left\|y-x^{\prime}(t)\right\|<\lambda\right\} .
$$

Since these diameters approach 0 as $\lambda \rightarrow 0$, we conclude $T\left(x_{k}, y_{k}\right) \rightarrow T(x, y)$ as $k \rightarrow \infty$ in this case.

In case $t_{0}=b$, the above argument can be modified slightly to obtain $T\left(x_{k}, y_{k}\right) \rightarrow T(x, y)$ as $k \rightarrow \infty$. Finally, consider the possibility $t_{0}=a$. We have $T(x, y)=x(a)+A_{2} x^{\prime}(a)$ and $r(x(a))=0$. By (6), the angle $\theta_{1}$, between $u(x(a))$ and $x(a)$ has $\cos \theta_{1} \geqslant \alpha$, and by (8), the angle $\theta_{2}$ between $x^{\prime}(a)$ and $x(a)=A_{1} x^{\prime}(a)$ has $\cos \theta_{2} \geqslant \sqrt{1-\alpha^{2}}$. Thus $\cos ^{2} \theta_{1}+\cos ^{2} \theta_{2} \geqslant 1$ and $\theta_{1}$ $+\theta_{2} \leqslant \pi / 2$. By a well-known result from geometry, the angle between $u(x(a))$ and $x^{\prime}(a)$ is less than or equal to $\theta_{1}+\theta_{2}$, so $u(x(a)) \cdot x^{\prime}(a) \geqslant 0$. It follows that there is a $\delta>0$ so that $r(x(t))>0$ for $a<t \leqslant t_{0}+\delta$, and the remainder of the earlier argument can again be modified to conclude $T\left(x_{k}^{\prime}, y_{k}\right) \rightarrow T(x, y)$ as $k \rightarrow \infty$. Thus $T$ is continuous on $\bar{V}$.

Define $H: R^{n} \rightarrow W$ by $H(y)=\left(A_{1} y, y\right)$. Note that $H$ is a homeomorphism. Let $U=H^{-1}(V)$. Then $U$ is bounded, open, nonempty and its boundary $\partial U \subseteq\left\{y: r\left(A_{1} y\right)=0\right\}$. We will calculate the Brouwer degree for the map $T \circ H: \bar{U} \rightarrow R^{n}$. See Schwartz [6] for a discussion of the Brouwer degree and its properties.

Consider the maps $\lambda T \circ H+(1-\lambda) I$ for $0 \leqslant \lambda \leqslant 1$ on $\bar{U}$, where $I$ is the identity. Let $y \in \partial U$ and suppose $\lambda T \circ H y+(1-\lambda) y=0$. If $\lambda \neq 0$, we have $T \circ H y=-((1-\lambda) / \lambda) y$, i.e., $\left(A_{1}+A_{2}\right) y=-((1-\lambda) / \lambda) y$. Note also that $r\left(A_{1} y\right)=0$ and $y \neq 0$. If $A_{2} y=0$, then $A_{1} y=-((1-\lambda) / \lambda) y$ contradicts (8). If $A_{2} y \neq 0$, then (8) and (9) together imply $y \cdot\left(A_{1}+A_{2}\right) y>0$, 
which contradicts $\left(A_{1}+A_{2}\right) y=-((1-\lambda) / \lambda) y$. If $\lambda=0$, then $y=0$, another contradiction. Thus the maps $\lambda T \circ H+(1-\lambda) I$ do not vanish on $\partial U$ for $0 \leqslant \lambda \leqslant 1$. The Brouwer degree $d[T \circ H, U, 0]$ exists, and by invariance of degree under homotopy, $d[T \circ H, U, 0]=d[I, U, 0]=1$ since $0 \in U$. Thus there is a $y \in U$ so that $T \circ H y=0$. This means that there is a solution $x(t)$ of (1) with $\left(x(a), x^{\prime}(a)\right) \in V$ and $T\left(x(a), x^{\prime}(a)\right)=x\left(t_{0}\right)+A_{2} x^{\prime}\left(t_{0}\right)=0$.

Suppose that $r\left(x\left(t_{0}\right)\right)=0$. Since $t_{0}>a, u\left(x\left(t_{0}\right)\right) \cdot x^{\prime}\left(t_{0}\right) \geqslant 0$. Let $\phi_{1}$ be the angle between $u\left(x\left(t_{0}\right)\right)$ and $x\left(t_{0}\right), \phi_{2}$ the angle between $x^{\prime}\left(t_{0}\right)$ and $A_{2} x^{\prime}\left(t_{0}\right)$ and $\phi_{3}$ the angle between $u\left(x\left(t_{0}\right)\right)$ and $x^{\prime}\left(t_{0}\right)$. Then $\phi_{3} \leqslant \pi / 2$, and by (6), (9), $\cos ^{2} \phi_{1}+\cos ^{2} \phi_{2}>1$, so $\phi_{1}+\phi_{2}<\pi / 2$. Now the angle between $A_{2} x^{\prime}\left(t_{0}\right)$ and $x\left(t_{0}\right)$ is no larger than $\phi_{1}+\phi_{2}+\phi_{3}$, the sum of which is less than $\pi$, contradicting $A_{2} x^{\prime}\left(t_{0}\right)=-x\left(t_{0}\right)$. Thus $r\left(x\left(t_{0}\right)\right) \neq 0$, and it follows that $t_{0}=b$. We conclude that $x$ is a solution of (1), (2), (3) with $r(x(t))<0$ for $a \leqslant t \leqslant b$.

Now suppose initial value problems of (1) do not necessarily have unique solutions. We show first that $y P(x) \cdot y \geqslant 0$ whenever $r(x)=0$ and $u(x) \cdot y$ $=0$. Suppose, on the contrary, that there is an $x$ with $r(x)=0$ and a $y \in R^{n}$ with $\|y\|=1, u(x) \cdot y=0$ and $y P(x) \cdot y<0$. For all constants $c, r^{\prime}(c y)$ $=u \cdot c y=0$, so by $(7)^{\prime}$,

$$
r_{f}^{\prime \prime}=c^{2} y P(x) \cdot y+u(x) \cdot f(t, x, c y)>0 .
$$

Choose $c$ large enough that $c^{2} / \phi(c) \geqslant\|u(x)\| /|y P(x) \cdot y|$. Then by condition $(\mathrm{N})$,

$$
|u(x) \cdot f(t, x, c y)| \leqslant\|u(x)\|\|f(t, x, c y)\| \leqslant\|u(x)\| \phi(c) \leqslant c^{2}|y P(x) \cdot y|,
$$

contradicting (10).

By Lemma 2.1 in [5], there is a $\mu>0$ so that if $x(t)$ satisfies $\left\|x^{\prime \prime}\right\| \leqslant \phi\left(\left\|x^{\prime}\right\|\right)$ and $r(x(t)) \leqslant 0$ for $t \in[a, b]$, then $\left\|x^{\prime}(t)\right\| \leqslant \mu(a \leqslant t \leqslant b)$. Define for all $(t, x) \in[a, b] \times R^{n}$,

$$
F(t, x, y)= \begin{cases}f(t, x, y), & \|y\| \leqslant \mu \\ f(t, x, \mu y /\|y\|), & \|y\|>\mu\end{cases}
$$

Fix $(x, y)$ so that $r(x)=0$ and $r^{\prime}(x, y)=0$. If $\|y\| \leqslant \mu$, then $r_{F}^{\prime \prime}-r_{f}^{\prime \prime}>0$ at $(x, y)$. If $\|y\|>\mu$, then

$$
\begin{aligned}
r_{F}^{\prime \prime} & =y P(x) \cdot y+u(x) \cdot f(t, x, \mu y /\|y\|) \\
& =\left(\|y\|^{2} / \mu^{2}\right)((\mu y /\|y\|) P(x) \cdot \mu y /\|y\|)+u \cdot f(t, x, \mu y /\|y\|) \\
& \geqslant(\mu y /\|y\|) P(x) \cdot \mu y /\|y\|+u \cdot f(t, x, \mu y /\|y\|)>0,
\end{aligned}
$$

since $r_{f}^{\prime \prime}>0$. Thus $F$ satisfies $(7)^{\prime}$.

Choose a sequence of $C^{1}$ functions $\left\{F_{n}\right\}_{n=1}^{\infty}$ which converges uniformly to $F$ on $[a, b] \times\{x: r(x) \leqslant 0\} \times R^{n}$. For $n$ sufficiently large, $F_{n}$ satisfies $(7)^{\prime}$ and 
condition (N), so we have a sequence $\left\{x_{n}\right\}$ so that each $x_{n}$ satisfies $x^{\prime \prime}$ $=F_{n}\left(t, x, x^{\prime}\right),(2),(3)$ and $r(x(t))<0$ for $t \in[a, b]$. Some subsequence of $\left\{x_{n}\right\}$ converges to a solution $x$ of $x^{\prime \prime}=F\left(t, x, x^{\prime}\right)$, (2), (3) with $r(x(t)) \leqslant 0$ for $a \leqslant t \leqslant b$. Since $F$ satisfies $\|F(t, x, y)\| \leqslant \phi(\|y\|)$ for $r(x(t)) \leqslant 0$ and $y \in R^{d}$, we have $\left\|x^{\prime}(t)\right\| \leqslant \mu$ for $t \in[a, b]$, so $x$ is a solution of (1).

Finally, if $f$ satisfies (7), then $f_{\varepsilon}=f+\varepsilon u(\varepsilon>0)$ satisfies (7)', so the above results apply to $f_{\varepsilon}$, and the proof is completed by letting $\varepsilon \rightarrow 0$. Q.E.D.

Of particular interest is the case that both $A_{1}$ and $A_{2}$ are linear. The assumption that $\left\{y: r\left(A_{1} y\right)<0\right\}$ is bounded requires $A_{1}$ to be nonsingular, and (8) requires, in addition, that for all $y \neq 0$, the angle between $y$ and $A_{1} y$ has cosine at least $\sqrt{1-\alpha^{2}}$. Note that if $y \in \operatorname{kernel} A_{2}$, then $y \notin D$. Thus (9) dictates that the cosine of the angle between $y$ and $A_{2} y$ is greater than $\sqrt{1-\alpha^{2}}$ for all $y \notin \operatorname{ker} A_{2}$. The most elementary choice for $r$ is $r(x)$ $=\|x\|^{2}-R^{2}$, where $R>0$. Then (7) becomes

$$
\|y\|^{2}+x \cdot f(t, x, y) \geqslant 0 \text { whenever }\|x\|=R \text { and } x \cdot y=0,
$$

and (6) is satisfied with $\alpha=1$, so one requires that $A_{1}$ be nonsingular and nonnegative definite and $A_{2} y \cdot y>0$ for all $y \notin \operatorname{ker} A_{2}$.

COROllary. Assume $A_{1}$ and $A_{2}$ are linear and $f$ satisfies condition $(\mathrm{N})$ with respect to $r(x)=\|x\|^{2}-R^{2}$ for some $R>0$. Suppose that (11) is satisfied, $A_{1}$ is nonsingular, $A_{1} y \cdot y \geqslant 0$ for all $y \in R^{n}$ and $A_{2} y \cdot y>0$ for all $y \notin \operatorname{ker} A_{2}$. Then there is at least one solution $x$ of (1), (2), (3) with $\|x(t)\| \leqslant R$ for $a \leqslant t \leqslant b$.

The next theorem provides criteria for the existence of a solution to (1), (4), (5). A proof can be given which is almost identical to the proof of Theorem 1 .

THEOREM 2. Let $r$ be as above and assume $\{x: r(x)<0\}$ is bounded and contains 0 . Suppose $u(x) \neq 0$ whenever $r(x)=0$, and $(6)$ is satisfied. Assume $f$ satisfies condition $(\mathrm{N})$ with respect to $r$ and $(7)$ holds. Suppose

$$
\begin{aligned}
\inf \left\{\left(x \cdot B_{1} x\right) /\|x\|\left\|B_{1} x\right\|: r(x)=0 \text { and } B_{1} x \neq 0\right\} \geqslant \sqrt{1-\alpha^{2}}, \\
\\
B_{2} x \neq 0 \text { whenever } r(x)=0 \text { and } \\
\quad \inf \left\{\left(x \cdot B_{2} x\right) /\|x\|\left\|B_{2} x\right\|: r(x)=0\right\}>\sqrt{1-\alpha^{2}}
\end{aligned}
$$

Then there is at least one solution $x$ of (1), (4), (5) with $r(x(t)) \leqslant 0$ for $t \in[a, b]$.

\section{REFERENCES}

1. J. W. Bebernes and R. Wihelmsen, $A$ general boundary value problem technique, J. Differential Equations 8(1970), 404-415. MR 42 \#575. 
2. L. K. Jackson and G. Klaasen, $A$ variation of the topological method of Waiewski, SIAM J. Appl. Math. 20 (1971), 124-130. MR 43 \# 5099.

3. A. Lasota and J. A. Yorke, Existence of solutions of two-point boundary value problems for nonlinear systems, J. Differential Equations 11 (1972), 509-518. MR 45 \#8915.

4. J. Mawhin, Boundary value problems for nonlinear second-order vector differential equations, J. Differential Equations 16 (1974), 257-269. MR 50 \# 13696.

5. K. Schmitt and R. Thompson, Boundary value problems for infinite systems of second-order differential equations, J. Differential Equations 18 (1975), no. 2, 277-295. MR 51 \# 10794.

6. J. T. Schwartz, Nonlinear functional analysis, Gordon and Breach, New York, 1969.

Department OF Mathematics, University OF OKLAHOMa, NORMan, OKLAHOMA 73069 